\title{
LES NOUVELLES DISPOSITIONS DU CODE RURAL SUR LES INTRODUCTIONS D'ESPĖCES ISSUES DE LA LOI DU 2 FÉVRIER 1995 RELATIVE AU RENFORCEMENT DE LA PROTECTION DE L'ENVIRONNEMENT.
}

\author{
THE NEW CLAUSES OF THE RURAL CODE REGARDING SPECIES \\ INTRODUCTION, STEMMING FROM THE LAW OF 2 FEBRUARY 1995 \\ THAT TIGHTENED ENVIRONMENTAL PROTECTION.
}

\begin{abstract}
J.P. GALLAND *
Chargé de la flore sauvage, Direction de la Nature et des Paysages, Ministère de I'Environnement, 20 avenue de Ségur, 75302 Paris 07 SP, France.
\end{abstract}

\section{INTRODUCTION}

Les effets néfastes des introductions d'espèces, volontaires ou accidentelles, sont de plus en plus fréquemment évoqués parmi les menaces qui pèsent sur les milieux naturels et les espèces sauvages indigènes, aux côtés d'autres atteintes plus " classiques " liées aux activités humaines. Le problème est connu de longue date dans les milieux insulaires (île de la Réunion, par exemple) mais le développement de cette problématique dans les milieux continentaux, sujet du présent colloque, semble plus récent. II n'est pourtant pas inutile de rappeler que les grands textes internationaux dans le domaine de la conservation des espèces sauvages comportent tous une disposition relative aux introductions d'espèces non indigènes :

- Directive " oiseaux " du 2 avril 1979, article 11 : " les Etats-membres veillent à ce que l'introduction éventuelle d'espèces d'oiseaux ne vivant pas naturellement à l'état sauvage sur le territoire européen des Etats-membres ne porte aucun préjudice à la flore et à la faune locales".

- Convention de Berne du 19 septembre 1979, article 11, paragraphe 2b) : " chaque Partie contractante s'engage à contrôler strictement l'introduction des espèces non indigènes ".

- Directive “ Habitats-Faune-Flore " du 21 mai 1992, article 22, alinéa b) : " les Etatsmembres veillent à ce que l'introduction intentionnelle dans la nature d'une espèce non indigène à leur territoire soit réglementée de manière à ne porter aucun préjudice aux habitats naturels dans leur aire de répartition naturelle ni à la faune et à la flore sauvages indigènes et, s'ils le jugent nécessaire, interdisent une telle introduction ".

- Convention de Rio sur la diversité biologique du 5 juin 1992, article 8, alinéa h) : " chaque Partie contractante, dans la mesure du possible et selon qu'il conviendra : empêche d'introduire, contrôle ou éradique les espèces exotiques qui menacent des écosystèmes, des habitats ou des espèces".

\footnotetext{
- Faisant suite à la disparition brutale de l'auteur, le présent article est livré en l'état, dans une forme qui n'a pu être totalement achevée. Déplorant ce triste concours de circonstances, nous remercions les lecteurs pour leur compréhension. 


\section{LES PROBLÈMES POSÉS PAR LES INTRODUCTIONS D'ESPĖCES}

Dans les textes internationaux qui viennent d'être rappelés, il n'est question que des espèces strictement exotiques, c'est-à-dire non représentées dans la faune et la flore du territoire considéré. Mais il ne faut pas oublier un autre aspect du problème, qui semble préoccuper tout autant la communauté scientifique : l'introduction dans la nature de spécimens appartenant à une espèce indigène, mais dont l'origine est inconnue ou lointaine. II convient d'examiner séparément ces deux questions, afin d'identifier les problèmes posés dans chaque cas.

\subsection{Introduction d'espèces exotiques}

Les risques sont liés au comportement envahissant que peut prendre une espèce introduite dans un milieu qui lui est favorable et où elle ne rencontre plus les facteurs limitants qui existaient dans son écosystème d'origine. II en résulte des phénomènes de compétition, voire d'élimination des espèces locales qui occupaient la même niche écologique. II s'agit d'un problème gènéral mais qui prend une acuité particulière dans les milieux insulaires, comme nous l'avons rappelé en introduction. Exemples d'espèces : Caulerpa taxifolia en Méditerranée, Carpobrotus edulis sur le littoral corse, tortue de Floride... Le déséquilibre écologique peut également être causé par le caractère prédateur de l'espèce introduite (exemple du “ blackbass à grande bouche ", au Portugal).

\subsection{Introduction de spécimens de provenance lointaine ou inconnue}

Les risques sont ici au niveau infraspécifique : flux de gènes entre population introduite et population autochtone. La population autochtone peut ainsi acquérir des caractères allant à l'encontre de son adaptation aux conditions locales (caractères de sensibilité, de domestication...) et pouvant aller jusqu'à causer sa disparition si le flux de gènes est massif. Exemple : les populations sauvages françaises de peuplier noir (Populus nigra) ont été très largement “ polluées » par les gènes provenant des cultivars utilisés en populiculture.

II convient de noter que le risque de "pollution génétique " existe également dans le cas des espèces exotiques (1.1.), qui peuvent s'hybrider avec des espèces indigènes proches. Les hybrides interspécifiques qui en résultent peuvent alors prendre un comportement envahissant. Exemple : Spartina anglica, hybride apparu entre une spartine indigène en France et une spartine américaine introduite, risque d'éliminer complètement son progéniteur européen.

\subsection{Les risques sanitaires}

Ils concernent à la fois les espèces et les populations non indigènes qui peuvent apporter de nouveaux pathogènes ou de nouveaux parasites causant des ravages dans la faune ou la flore locales. Les exemples sont nombreux chez les poissons (sandre introduit de Hongrie...).

\section{LES OBJECTIFS POURSUIVIS PAR LE MINISTĖRE DE L'ENVIRONNEMENT}

"Les espèces animales et végétales, la diversité et les équilibres biologiques auxquels elles participent font partie du patrimoine commun de la nation " (art. L.200-1 nouveau du Code rural). Les autorités en charge de la protection de la nature doivent donc veiller à :

- prévenir l'altération du patrimoine biologique qui résulterait d'introductions du fait de l'activité humaine (et non du fait de phénomènes naturels d'extension d'aire ou de dissémination) ;

- contrôler les effets des introductions qui se sont avérées dommageables pour la faune et la flore sauvages.

Pour parvenir à ces fins, les autorités doivent mettre en oeuvre, dans une politique cohérente, des outils réglementaires ou incitatifs qui devront être accompagnés d'actions d'information. 


\section{LA LOI “BARNIER »ET SON APPLICATION}

Nous n'aborderons pas ici les dispositions juridiques relatives au contrôle de l'importation de certaines espèces sur le territoire français ou sur le territoire communautaire et nous limiterons au problème précis de l'introduction dans la nature. Jusqu'à la loi du 2 février 1995, dite "Loi Barnier", seules les espèces de poissons (crustacés et grenouilles) bénéficiaient d'une réglementation de leur introduction issue de la loi sur la pêche. La loi du 2 février 1995 relative au renforcement de la protection de l'environnement a introduit deux nouveaux articles (L.211-3 et L.211-4) dans le Code rural, dont la portée est volontairement très générale afin de couvrir tous les types de situations. L'article L.211-3 énonce un principe général d'interdiction pour l'introduction dans le milieu naturel :

- de tout spécimen d'une espèce animale, à la fois non indigène au territoire d'introduction et non domestique ;

- de tout spécimen d'une espèce végétale, à la fois non indigène au territoire d'introduction et non cultivée:

- de tout spécimen de l'une des espèces animales ou végétales désignées par l'autorité administrative.

La loi prévoit ensuite une possibilité d'autorisation d'introduction délivrée par l'autorité administrative (par dérogation au principe d'interdiction) à des fins agricoles, piscicoles, forestières ou pour des motifs d'intérêt général, et après évaluation des conséquences prévisibles de l'introduction. Le décret d'application, actuellement en cours de préparation, va devoir définir la procédure d'autorisation d'introduction et délimiter le champ d'application de ces mesures. En effet, le texte de la loi utilise des expressions qui font partie du langage commun mais dont les limites peuvent donner lieu à bien des débats scientifiques et juridiques, comme par exemple :

- milieu naturel : I'idée sous-jacente est simplement de considérer qu'il ne serait ni réaliste, ni justifié, de vouloir contrôler les introductions dans des milieux "confinés ", dans lesquels l'espèce considérée reste contrôlable;

- espèce non indigène : le problème se pose pour les espèces d'apparition occasionnelle, et surtout pour les espèces anciennement introduites qui se sont acclimatées : faut-il fixer une limite de date pour considérer qu'une espèce est devenue indigène et, si oui, laquelle?

- territoire d'introduction : le choix de limites biogéographiques pour définir un territoire d'introduction d'une espèce serait certainement plus satisfaisant sur le plan scientifique mais poserait d'épineux problèmes d'application. II semble plus raisonnable de se contenter de limites administratives plus simples, et qui d'ailleurs avaient été évoquées lors des débats préalables au vote de la loi, c'est-à-dire de considérer comme territoire d'introduction : le territoire continental de la France métropolitaine, le domaine marin atlantique, le domaine marin méditerranéen, la Corse, et chacun des départements et des collectivités territoriales d'outremer.

Le droit de l'environnement est, par nature, un droit très technique et les autorités chargées de préparer ce décret sur les introductions d'espèces se trouvent, une fois de plus, condamnées à trouver un compromis (le moins mauvais possible) entre deux solutions extrêmes : donner des définitions scientifiques très précises aux notions figurant dans la loi, au risque de faire de cette réglementation une affaire de spécialistes, ou bien laisser au Juge une marge d'appréciation au cas par cas, au risque de laisser le citoyen dans l'incertitude quant à la légalité de ses actes.

Rappelons que le but de toute cette démarche est de contrôler, par un système d'autorisation administrative, les introductions volontaires dans le milieu naturel. Cependant, il ne faut pas oublier que, dans la plupart des cas, les espèces qui ont causé des dommages à 
la faune et à la flore sauvages n'avaient pas été introduites volontairement. II s'agit plutôt d'introductions accidentelles: spécimens échappés (de captivité ou de cultures) ou arrivés clandestinement à la faveur du transport de certains produits ou matériaux (les eaux de ballast des navires en sont un exemple typique). Dans ce contexte, une lourde mécanique administrative de contrôle des introductions volontaires (si tant est qu'elle puisse être mise en place) risquerait donc d'être à la fois démesurée et insuffisante au regard des vrais problèmes.

Les dispositions issues de la loi "Barnier" auront néanmoins le grand mérite de responsabiliser les citoyens en permettant de sanctionner, tant sur le plan pénal que sur le plan civil, le responsable d'une introduction préjudiciable aux milieux naturels ou à la faune et à la flore sauvages, et ce même s'il s'agit d'une introduction involontaire résultant de sa négligence ou de son imprudence. En effet, l'introduction volontaire d'une espèce interdite par la loi est constitutive d'un délit, en application de l'article L.215-1 du Code rural. Le fait de laisser introduire une telle espèce par négligence ou par imprudence sera, par ailleurs, passible des peines prévues pour les contraventions de la 4ème classe.

Pour terminer, mentionnons encore deux remarques touchant l'application des nouveaux articles L.211-3 et L.211-4 du Code rural :

1) Ces dispositions couvrent surtout le problème des espéces exotiques. Les problèmes liés à l'introduction de spécimens d'espèces indigènes, et à la prévention des risques d'introgression que nous avons mentionnés plus haut, ne sont pas abordés directement. Cependant, la loi permet $d^{\prime}$ inscrire sur la liste prévue au $3^{\circ}$ de l'article L.211-3, les espèces (et pas seulement les espèces sauvages) dont on souhaite contrôler l'introduction dans la nature. II pourrait être envisagé, par exemple, que les espèces protégées soient soumises à ce régime afin de contrôler plus directement les projets de renforcements de population. II en va de même pour d'autres types d'espèces, comme les espèces gibier.

2) Le contrôle des espèces déjà introduites et causant des dommages est peu abordé par la loi. Le décret d'application cherchera à préciser la question des mesures à prendre face à une espèce introduite portant préjudice aux milieux naturels, à la faune ou à la flore sauvages.

\section{CONCLUSION}

Une étude réalisée par Cyrille de KLEMM pour le compte du Conseil de l'Europe a montré que peu de pays disposaient de mesures juridiques de contrôle des introductions d'espèces exotiques couvrant toutes les espèces, comme c'est aujourd'hui le cas en France.

La préparation du décret d'application de la loi "Barnier " donnera certainement lieu à des débats interministériels difficiles mais, une fois le dispositif en place, la France pourra se réjouir d'une avancée juridique et d'une application opportune du principe de précaution. 